CLINICAL STUDY

\title{
Anaplastic thyroid carcinoma: clinical outcome of 30 consecutive patients referred to a single institution in the past 5 years
}

\author{
Enrico Brignardello ${ }^{1}$, Marco Gallo ${ }^{1}$, Ileana Baldi ${ }^{2}$, Nicola Palestini ${ }^{3}$, Alessandro Piovesan ${ }^{1}$, Emidio Grossi $^{1}$, \\ Giovannino Ciccone ${ }^{2}$ and Giuseppe Boccuzzi ${ }^{1,4}$ \\ ${ }^{1}$ Oncological Endocrinology Unit and ${ }^{2}$ Cancer Epidemiology Unit, San Giovanni Battista Hospital, Turin, Italy, Departments of ${ }^{3}$ Surgery and ${ }^{4}$ Clinical \\ Pathophysiology, University of Turin, Turin, Italy
}

(Correspondence should be addressed to G Boccuzzi who is now at Department of Clinical Pathophysiology, Via Genova, 3, 10126 Turin, Italy;

Email: giuseppe.boccuzzi@unito.it)

\begin{abstract}
Objective: Treatment options for anaplastic thyroid carcinoma (ATC), which is one of the most lethal human malignant tumors, include surgery, chemotherapy and radiotherapy usually combined in a multimodal approach, to improve survival and avoid death from local invasion. However, there is no standard protocol for ATC treatment and the optimal sequence within multimodal therapy is debated. We retrospectively report the clinical outcome of 30 ATC patients referred consecutively to the Oncological Endocrinology Unit of San Giovanni Battista Hospital (Turin, Italy) between 2000 and 2005.

Design: Patients were treated by one of the following approaches: i) surgery followed by adjuvantcombined chemoradiotherapy; ii) neo-adjuvant chemoradiotherapy followed by surgery and adjuvant chemotherapy; or iii) chemotherapy alone. The surgical procedures were classified as 'maximal debulking' or 'palliative resection'. Maximal debulking entailed total or near-total thyroidectomy and complete resection of all gross tumor or minimal residual disease adherent to vital structures, independently of the presence or absence of distant metastases. In palliative resections, macroscopic residual disease was left in the neck. Survival of patients stratified by treatment was assessed.

Results: Analysis of multivariate hazard ratios showed that maximal debulking followed by adjuvant chemoradiotherapy was the only treatment that modified survival of ATC patients (hazard ratio $=$ 0.23, 95\% CI: 0.07-0.79), even if factors determining poor prognosis or increased surgical risk were present.

Conclusions: Despite the overall grim outcome of ATC, these results justify an attempt at maximal debulking surgery, followed by adjuvant chemoradiotherapy, possibly in all ATC patients.

European Journal of Endocrinology 156 425-430
\end{abstract}

\section{Introduction}

Anaplastic thyroid carcinoma (ATC) is a rare disease, accounting for $<2 \%$ of all thyroid tumors (1). Nevertheless, it is one of the most lethal human malignant tumors, accounting for about one-half of all thyroid carcinoma deaths (2-4). ATC usually presents as a rapidly enlarging neck mass, often associated with vocal cord paralysis, dyspnea and/or dysphagia, with an F:M ratio of 1.5:1 and a peak of incidence in the sixth and seventh decades of life $(5,6)$. In stark contrast to the behavior of well-differentiated (papillary or follicular) thyroid cancer, ATC typically follows a rapid and lethal clinical course, with median survival below 6 months in the majority of series reported.

The disease is usually far advanced at the time of diagnosis, with extensive local disease and direct invasion into adjacent organs, such as the trachea, esophagus, blood vessels and muscle, in a high percentage of patients (7). Furthermore, about $50 \%$ of ATC patients present with distant metastases, the lung being the most commonly involved site $(4,8)$. Patients usually die from local and regional progression of the tumor, with airway and esophageal obstruction.

The options for treatment of ATC include surgery, chemotherapy and radiotherapy. All of these, especially if used alone, most often fail to control local disease. Multimodal therapy, combining surgery, chemotherapy and radiation therapy, can give better results, avoiding death from local invasion and suffocation and improving survival of some ATC patients (8-11). Nevertheless, the aggressive nature and rarity of ATC makes it difficult to compare patient outcomes, especially in studies with small cohorts and short follow-up. Therefore, a 
standardized successful protocol is lacking and the optimal sequence of multimodal therapy is still debated $(12,13)$.

Since local control is a leading component in treating ATC patients, it has been proposed that - in a multimodal therapy context - surgical debulking followed by adjuvant combined chemoradiotherapy represents the best option, since a smaller tumor burden remains to be treated by radiation and chemotherapeutic agents (11, $14,15)$. By contrast, others indicate a regimen consisting of neo-adjuvant radiotherapy combined with chemotherapy (as radiosensitizing agent), followed by surgery as the best treatment for ATC (10).

Here, we retrospectively report the clinical outcome of all ATC patients treated in our Institution between 2000 and 2005.

\section{Subjects and methods}

We considered all consecutive cases of pure ATC referred to the Oncological Endocrinology Unit of San Giovanni Battista Hospital (Turin, Italy) from the second half of 2000 through to 2005.

Diagnosis of ATC was obtained by cytological specimens derived from fine needle aspiration and confirmed by histological examination in all patients who underwent surgery. Differentiated or poorly differentiated thyroid carcinomas with focal areas of anaplastic cancer were not included in this series. All smears were reviewed by a single pathologist to confirm the diagnosis of ATC. Patients were treated by one of the following approaches:

(i) When feasible, patients were first operated and subsequently treated by combined chemotherapy and radiotherapy. The surgical procedures were classified as 'maximal debulking' or 'palliative resection'. Maximal debulking entailed total or near-total thyroidectomy and complete resection of all gross tumor or minimal residual disease adherent to vital structures (pharynx, esophagus, larynx or trachea), independently of the presence or absence of distant metastases. Any other operation leaving more than minimal residual disease in the neck was considered a palliative resection. Depending on the situation, in several cases maximal debulking required en bloc resection of adjacent muscles, internal jugular vein, and/or recurrent laryngeal nerve (RLN) involved by the tumor; at least one RLN was always preserved. Extensive neck operations with resection of the esophagus, trachea, or larynx are not warranted for this tumor, and in no case were they performed.

Radiotherapy was administered once daily, 5 days a week, with a target dose of 1.8-2.0 Gy (i.e. total target dose of 36-40 Gy delivered over
4 weeks). Chemotherapy consisted of doxorubicin $(20 \mathrm{mg} / \mathrm{mq})+$ cis-platin $(20 \mathrm{mg} / \mathrm{mq})$, starting $1-2 \mathrm{~h}$ before the first fraction and administered once weekly for the entire duration of radiotherapy. After completion of radiotherapy, chemotherapy was continued with doxorubicin $(60 \mathrm{mg} / \mathrm{mq})+$ cis-platin $(50-60 \mathrm{mg} / \mathrm{mq})$, administered every 3 weeks, up to a maximum doxorubicin dose of $550 \mathrm{mg} / \mathrm{mq}$ or until tolerated. Patients who were still alive after completion of first-line chemotherapy (and with evidence of progressive disease) underwent second-line chemotherapy with paclitaxel $80-120 \mathrm{mg} / \mathrm{mq}$ every week, for 6 consecutive weeks out of 8 weeks (16).

(ii) Patients who were judged not operable by the surgeon at the time of referral received radiotherapy combined with radiosensitizing chemotherapy (doxorubicin $(20 \mathrm{mg} / \mathrm{mq})+$ cis-platin (20 mg/mq) once weekly for the entire duration of radiotherapy), administered with neo-adjuvant intent. When feasible, surgery was performed about 3 weeks after completion of the combined chemoradiotherapy and followed by adjuvant chemotherapy (doxorubicin $(60 \mathrm{mg} / \mathrm{mq})+$ cisplatin $(60 \mathrm{mg} / \mathrm{mq})$ every 3 weeks, up to a total doxorubicin dose of $550 \mathrm{mg} / \mathrm{mq}$ ).

(iii) In the presence of contraindications to both surgery and radiotherapy, patients were treated by chemotherapy alone. In this case, we used paclitaxel as a single agent, administered weekly at a dose of $80-120 \mathrm{mg} / \mathrm{mq}$, for 6 consecutive weeks out of 8 weeks. This took into account the established low efficacy of platinum and doxorubicin when not associated with radiotherapy and reports of the effectiveness of paclitaxel against human ATC, both in experimental models and in ATC patients (16-18).

Patients who did not receive any treatment beyond palliation were excluded from the analysis of survival by treatment. For the purpose of the analyses, neck surgery was classified into three categories: maximal debulking, palliative resection and none.

The time-axis we considered for survival analysis was months after ATC diagnosis. The follow-up was updated as of December 31, 2005. Crude KaplanMeier survival probabilities were estimated both overall and stratified by several variables. Differences between curves were tested using the two-tailed log-rank test. Because survival curves are known to be unstable in the tail (19), they were truncated at 12 months since diagnosis.

Univariate and multivariate hazard ratios (HR) and corresponding 95\% confidence intervals $(95 \% \mathrm{CI})$ were estimated via the Cox proportional hazard model. Given the small sample size, choice of the covariates to include in the multivariate Cox model was based on both 
clinical and statistical considerations. As a starting point, we included a known prognostic factor (presence of metastases at diagnosis) and other variables were then selected following the Akaike Information Criterion (AIC; 20). The concept underlying the AIC is to examine the complexity of the model together with its goodness-of-fit to the sample data and to produce a measure balancing the two. The usual procedure is to compute the AIC for each model and select that corresponding to the lowest value.

We checked proportionality of hazard rates by Grambsh and Therneau tests (21). We also validated this model using the bootstrap on 100 samples. A $P$ value of 0.05 or less was considered statistically significant. R software, v.2.1.0 (package Design) was used (22).

\section{Results}

During the interval of time considered, 30 patients were referred to our institution. The majority were women (2:1). The age range was 46-92 years, with peak incidence in the sixth and seventh decades of life. The mean size of the tumor at the time of diagnosis was $6.0 \pm 2.1 \mathrm{~cm}$ (mean \pm s.D.). At diagnosis, tracheal and esophageal infiltration was observed in 11 and 8 patients respectively. Distant metastases, most common at the lungs, were found at the time of diagnosis in 15 patients.

Median survival was 3.9 months. Three patients were excluded from statistical analysis, two patients because they did not receive any treatment beyond palliation (due to extreme old age and poor general condition), and one patient because ATC was a small focus $(3 \mathrm{~mm})$ incidentally discovered after surgery for compressive goiter. The two patients who received only palliative therapy died within a few weeks, whereas the patient with an incidentally discovered small focus of ATC is still alive and disease free. Overall survival of treated patients at 6 months was 35.7\% (95\% CI: 20.6$62.0 \%$ ) as shown in Fig. 1. Baseline characteristics of eligible patients, stratified according to the type of intervention, are shown in Table 1.

Almost $75 \%$ of all patients with ATC underwent surgical resection. Among patients who were operated initially, 11 patients underwent maximal debulking and 6 patients underwent palliative resection. Fifteen of these patients were subsequently treated by adjuvantcombined chemoradiotherapy, as described earlier. Only five patients in this group died from local progression of the disease.

Of the five patients treated with combined chemoradiotherapy administered with neo-adjuvant intent, only in one case was it possible to complete the treatment schedule by surgery and adjuvant chemotherapy. This patient showed a complete response to treatment and is alive and well, without any evidence of

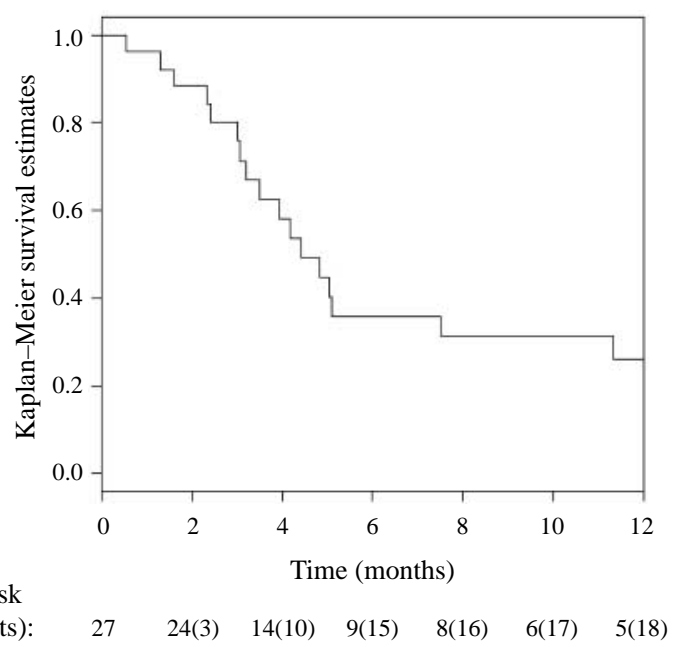

Figure 1 Overall survival according to crude Kaplan-Meier estimates.

disease 34 months after surgery. Unfortunately, the other four patients had a worse outcome: two patients died before surgery, for local progression of the disease, and the other two patients shortly afterwards.

The overall outcome of the five patients treated with chemotherapy alone was dismal. The median survival was 3.2 months, local progression also being the cause of death for these patients.

Crude survival was similar in patients treated by palliative resection and in those not operated at all (Fig. 2). Due to this similar outcome, neck surgery was dichotomized as 'maximal debulking' versus 'palliative resection or none'.

At univariate analysis, presence of metastases $(P=0.007)$ and type of intervention $(P<0.001)$ were the most important prognostic factors, whereas sex, age at diagnosis, primary tumor size, esophageal and tracheal infiltration were less predictive (Table 2). The final Cox model derived from our selection (corresponding $\mathrm{AIC}=79.6$ ) included presence of metastases, type of neck surgery and tracheal infiltration. The proportionality assumption was satisfied overall (Grambsh and Therneau $P=0.96$ ) and the overfitting corrected $R^{2}$ was 0.34 . This multivariate analysis confirmed that presence of metastases and types of intervention were the most relevant prognostic factors. Patients treated by maximal debulking surgery showed a significantly lower risk of death than patients who underwent palliative resection or who were not operated at all (HR $=0.23$, 95\% CI: 0.07-0.79), without any significant complication related to treatment, even after adjusting for the presence of distant metastases and tracheal infiltration at diagnosis.

Alternative model specifications, which included either tracheal or esophageal infiltration (rather than 
Table 1 Patients' baseline characteristics stratified by surgical approach.

\begin{tabular}{|c|c|c|c|c|}
\hline \multirow[b]{2}{*}{ Baseline characteristics } & \multirow[b]{2}{*}{ Total sample $(n=27)$} & \multicolumn{3}{|c|}{ Surgical approach } \\
\hline & & Maximal debulking $(n=14)$ & Palliative resection $(n=6)$ & None $(n=7)$ \\
\hline \multicolumn{5}{|l|}{ Sex } \\
\hline $\mathrm{F}: \mathrm{M}$ & $18: 9$ & $12: 2$ & $3: 3$ & $3: 4$ \\
\hline \multicolumn{5}{|l|}{ Age at diagnosis } \\
\hline Mean \pm S.D. & $69.8 \pm 10.2$ & $70.0 \pm 9.5$ & $71.6 \pm 11.3$ & $67.8 \pm 12.1$ \\
\hline Range & $46.0-88.8$ & $57.2-88.8$ & $56.5-81.1$ & $46.0-79.0$ \\
\hline \multicolumn{5}{|l|}{ Tumor size $(\mathrm{cm})$} \\
\hline Mean \pm S.D. & $6.1 \pm 1.8$ & $6.1 \pm 2.0$ & $6.0 \pm 1.7$ & $6.4 \pm 1.7$ \\
\hline Range & $2.5-9.0$ & $2.5-9.0$ & $4.0-8.7$ & $3.0-8.0$ \\
\hline Unknown & 2 & 1 & - & 1 \\
\hline \multicolumn{5}{|l|}{ Distant metastases } \\
\hline Yes & 15 & 7 & 4 & 4 \\
\hline No & 11 & 7 & 2 & 2 \\
\hline Unknown & 1 & - & - & 1 \\
\hline \multicolumn{5}{|l|}{ Tracheal infiltration } \\
\hline Yes & 11 & 5 & 3 & 3 \\
\hline No & 15 & 8 & 3 & 4 \\
\hline Unknown & 1 & 1 & - & - \\
\hline \multicolumn{5}{|l|}{ Esophageal infiltration } \\
\hline Yes & 8 & 5 & 1 & 2 \\
\hline No & 18 & 8 & 5 & 5 \\
\hline Unknown & 1 & 1 & - & - \\
\hline
\end{tabular}

tracheal infiltration alone) and age (corresponding AIC $=84$ ), confirmed the positive prognostic role of maximal debulking surgery $(\mathrm{HR}=0.19 ; 95 \%$ CI: 0.06-0.62).

In a subgroup analysis limited to the 20 surgical patients, the protective effect of maximal debulking versus palliative resection was confirmed and still statistically significant ( $\mathrm{HR}=0.21,95 \% \mathrm{CI}$ : 0.05-0.82).

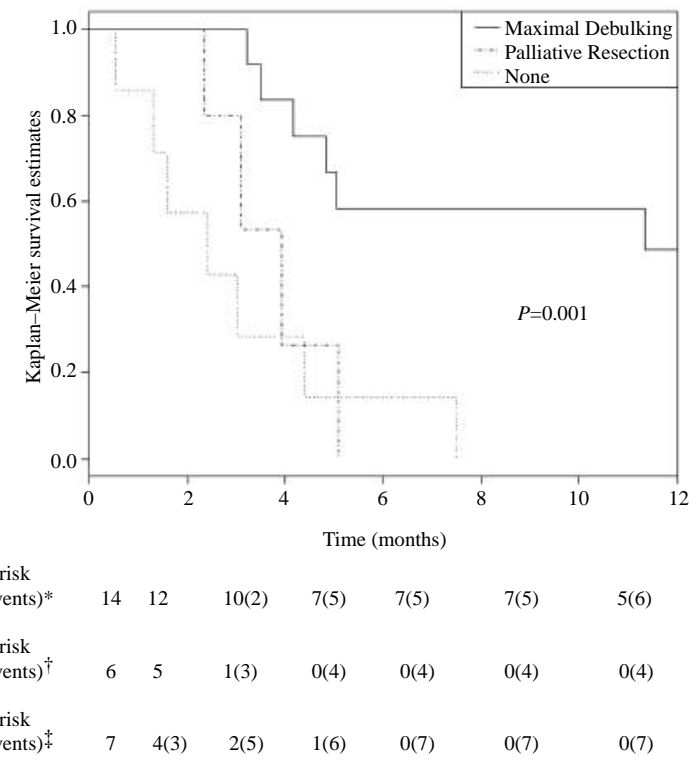

Figure 2 Survival stratified by neck surgery (maximal debulking*, palliative resection ${ }^{\dagger}$, or none ${ }^{\ddagger}$ ) according to Kaplan-Meier estimates.

\section{Discussion}

Despite its rarity, anaplastic thyroid carcinoma is one of the leading causes of death in oncological endocrinology. The retrospective analysis of the cases referred to our institution identifies maximal debulking surgery followed by adjuvant chemoradiotherapy as the treatment which most improves clinical outcome. A prolonged survival by means of an aggressive surgical approach, followed by adjuvant chemoradiotherapy, has already been reported $(14,15,23)$. Survival of patients treated in this way was significantly better than that of patients who underwent palliative resection or who were not operated at all, even after adjusting for tracheal infiltration and presence of metastases.

It is well known that a major confounding factor in determining the effect of treatment on outcome is selection bias, since patients who undergo maximal debulking surgery could have a better prognosis due to less extensive disease. According to World Health Organization definitions, differentiated or poorly differentiated thyroid carcinomas with focal areas of ATC should be diagnosed as anaplastic cancer. However, we did not include these cases in our series in order to increase homogeneity among patients. In our sample, some preoperative clinical features, such as age at diagnosis, size of primary tumor and presence of tracheal infiltration, were similar in patients who underwent palliative resection and in those subjected to maximal debulking surgery. It should be noted that most palliative resections were performed at first-referral centers, possibly by surgeons with inadequate experience in this field and sometimes unaware of the diagnosis. 
Table 2 Overall survival of the 27 anaplastic thyroid carcinoma patients.

\begin{tabular}{|c|c|c|c|}
\hline \multirow[b]{2}{*}{ Variables } & \multirow[b]{2}{*}{$\begin{array}{c}\text { Kaplan-Meier estimates: } \\
\% 6 \text { months survival }(95 \% \mathrm{Cl})\end{array}$} & \multicolumn{2}{|c|}{ Cox models } \\
\hline & & Univariate HR $(95 \% \mathrm{Cl})$ & Multivariate $\mathrm{HR}(95 \% \mathrm{Cl})$ \\
\hline \multicolumn{4}{|l|}{ Metastases } \\
\hline No & $66.7(28.1-87.8)$ & 1 & 1 \\
\hline Yes & $15.6(2.5-39.1)$ & 3.89 (1.35-11.2) & $3.48(1.2-10.01)$ \\
\hline \multicolumn{4}{|l|}{ Neck surgery } \\
\hline Palliative or none & $10.3(0.6-36.2)$ & 1 & 1 \\
\hline Maximal debulking & $58.3(27.0-80.1)$ & $0.18(0.06-0.54)$ & $0.23(0.07-0.79)$ \\
\hline \multicolumn{4}{|l|}{ Tracheal infiltration } \\
\hline No & $43.1(17.9-66.2)$ & 1 & 1 \\
\hline Yes & $13.3(0.7-44.1)$ & $2.10(0.78-5.69)$ & $1.42(0.45-4.44)$ \\
\hline \multicolumn{4}{|l|}{ Sex } \\
\hline $\mathrm{F}$ & $40.0(16.5-62.8)$ & 1 & \\
\hline M & $27.8(4.4-59.1)$ & $1.55(0.62-3.92)$ & \\
\hline \multicolumn{4}{|l|}{ Age at diagnosis } \\
\hline$<70$ Years & $54.8(22.2-78.7)$ & 1 & \\
\hline$\geq 70$ Years & $23.2(5.6-47.7)$ & $2.41(0.89-6.55)$ & \\
\hline Tumor size $(\mathrm{cm})$ & - & $0.98(0.75-1.30)$ & \\
\hline \multicolumn{4}{|l|}{ Esophageal infiltration } \\
\hline No & 38.5 (16.0-60.8) & 1 & \\
\hline Yes & $17.1(0.8-52.6)$ & $1.29(0.49-3.42)$ & \\
\hline
\end{tabular}

Kaplan-Meier survival (\% at 6 months) and Cox survival models. Hazard ratios (HR) and 95\% confidence intervals (Cl).

In general, our sample confirms the previously reported sex and age distribution $(4-6,8,13)$. The overall median survival of 3.9 months is in accordance with some previous reports $(5,10,24-26)$, whereas it appears worse than other published results $(1,2,27)$. One explanation could be our more rigorous patient selection, excluding mixed forms of anaplastic and poorly differentiated tumors, as well as small anaplastic cancers of incidental finding.

Presence of distant metastases at diagnosis, most often involving the lung, was a common finding in our series. In accordance with findings of other studies $(6-8,28)$, this factor is associated with poorer survival. Tracheal or esophageal infiltration also seems to negatively affect the prognosis of these patients, but these associations are not statistically significant.

In our experience, patients treated by chemoradiotherapy administered with neo-adjuvant intent had a poor outcome; four out of the five patients receiving this approach could not complete the planned treatment (i.e. neo-adjuvant chemoradiotherapy, surgery, adjuvant chemotherapy) and died either before surgery or immediately afterwards. The outcome of patients treated by chemotherapy alone was also disappointing, but in agreement with other reports, showing that the currently available chemotherapeutic agents have little effect in the treatment of $\operatorname{ATC}(7,10$, $14,15)$. Indeed, when the tumor burden is large, it may be extremely difficult to counteract the very rapid growth of ATC with chemotherapy and radiotherapy.

A comparison between the effectiveness of different therapeutic approaches to ATC is clearly outside the scope (and the possibilities) of this observational study. Nonetheless, it should be emphasized that - in our experience - maximal debulking surgery followed by adjuvant chemoradiotherapy was the only treatment that modified the survival of ATC patients, independently of the presence of negative prognostic factors.

Such a complex and expensive approach might not be justified for a disease that remains almost universally and rapidly fatal. Nevertheless, most published studies include in their series some 'long' survivors, and six of our patients survived for more than 1 year. This result, in addition to the low incidence of perioperative complications, might justify an attempt at maximal debulking surgery followed by adjuvant-combined chemoradiotherapy for all ATC patients.

Further, multicentric studies are needed to confirm the usefulness of this approach. Nevertheless, the prognosis of ATC remains dismal and it is to be hoped that innovative strategies may be developed to tackle this lethal condition.

\section{References}

1 Gilliland FD, Hunt WC, Morris DM \& Key CR. Prognostic factors for thyroid carcinoma. A population-based study of 15698 cases from the surveillance, epidemiology and end results (SEER) Program 1973-1991. Cancer 199779 564-573.

2 Hundahl SA, Fleming ID, Fremgen AM \& Menck HR. A national cancer data base report on 53856 cases of thyroid carcinoma treated in the US. Cancer $1998 \mathbf{8 3} 2638-2648$.

3 Kitamura Y, Shimizu K, Nagahama M, Sugino K, Ozaki O, Mimura T, Ito K, Ito $\mathrm{K} \&$ Tanaka S. Immediate causes of death in thyroid carcinoma: clinicopathological analysis of 161 fatal cases. Journal of Clinical Endocrinology and Metabolism $1999 \mathbf{8 4}$ 4043-4049.

4 Are C \& Shaha AR. Anaplastic thyroid carcinoma: biology, pathogenesis, prognostic factors and treatment approaches. Annals of Surgical Oncology 200613 453-464. 
5 McIver B, Hay ID, Giuffrida DF, Dvorak CE, Grant CS, Thompson GB, van Heerden JA \& Goellner JR. Anaplastic thyroid carcinoma: a 50-year experience at a single institution. Surgery $20011301028-1034$.

6 Kebebew E, Greenspan FS, Clark OH, Woeber KA \& McMillan A. Anaplastic thyroid carcinoma. Treatment outcome and prognostic factors. Cancer 2005103 1330-1335.

7 Ain KB. Anaplastic thyroid carcinoma: behavior, biology and therapeutic approaches. Thyroid $1998 \mathbf{8}$ 715-726.

8 Pasieka JL. Anaplastic thyroid cancer. Current Opinion in Oncology 200315 78-83.

9 Ain KB. Anaplastic thyroid carcinoma: a therapeutic challenge. Seminars in Surgical Oncology 199916 64-69.

10 Tennvall J, Lundell G, Wahlberg P, Bergenfelz A, Grimelius L, Akerman M, Hjelm Skog AL \& Wallin G. Anaplastic thyroid carcinoma: three protocols combining doxorubicin, hyperfractionated radiotherapy and surgery. British Journal of Cancer $2002 \mathbf{8 6}$ $1848-1853$.

11 De Crevoisier R, Baudin E, Bachelot A, Leboulleux S, Travagli JP, Caillou B \& Schlumberger M. Combined treatment of anaplastic thyroid carcinoma with surgery, chemotherapy and hyperfractionated accelerated external radiotherapy. International Journal of Radiation Oncology, Biology, Physics $2004601137-1143$.

12 Vini L \& Harmer C. Management of thyroid cancer. Lancet Oncology 20023 407-414.

13 Kihara M, Miyauchi A, Yamauchi A \& Yokomise H. Prognostic factors of anaplastic thyroid carcinoma. Surgery Today 200434 394-398.

14 Haigh PI, Ituarte PHG, Wu HS, Treseler PA, Posner MD, Quivey JM, Duh QY \& Clark OH. Completely resected anaplastic thyroid carcinoma combined with adjuvant chemotherapy and irradiation is associated with prolonged survival. Cancer $2001912335-2342$.

15 Sugino K, Ito K, Mimura T, Nagahama M, Fukunari N, Kubo A, Iwasaki $\mathrm{H} \&$ Ito $\mathrm{K}$. The important role of operations in the management of anaplastic thyroid carcinoma. Surgery 2002131 245-248.

16 Ain KB, Egorin MJ \& DeSimone PA. Treatment of anaplastic thyroid carcinoma with paclitaxel: phase 2 trial using ninety-sixhour infusion. Collaborative anaplastic thyroid cancer health intervention trials (CATCHIT) Group. Thyroid 200010 587-594.

17 Voigt W, Kegel T, Weiss M, Mueller T, Simon H \& Schmoll HJ. Potential activity of paclitaxel, vinorelbine and gemcitabine in anaplastic thyroid carcinoma. Journal of Cancer Research and Clinical Oncology 2005131 585-590.
18 Ain KB, Tofiq S \& Taylor KD. Antineoplastic activity of taxol against human anaplastic thyroid carcinoma cell lines in vitro and in vivo. Journal of Clinical Endocrinology and Metabolism $1996 \mathbf{8 1}$ 3650-3653.

19 Marubini E \& Valsecchi MG. Analysing Survival Data from Clinical Trials and Observational Studies Chichester, New York: Wiley, 1995.

20 Akaike H. Information theory as an extension of the maximum likelihood principle. In Proceedings of the 2nd International Symposium on Information Theory, pp. 267-281. Eds B-N Petrov \& F Csaki, Budapest: Akademiai Kiado, 1973.

21 Therneau TM \& Grambsch PM. Modeling Survival Data: Extending the Cox Model, pp. 69-95, New York: Springer, 2000.

22 R Development Core Team. R: A language and environment for statistical computing. Vienna, Austria: R Foundation for Statistical Computing, 2005 (www.R-project.org).

23 Tennvall J, Lundell G, Hallquist A, Wahlberg P, Wallin G \& Tibblin S. Combined doxorubicin, hyperfractionated radiotherapy, and surgery in anaplastic thyroid carcinoma. Report on two protocols. The Swedish Anaplastic Thyroid Cancer Group. Cancer $1994741348-1354$.

24 Nilsson O, Lindeberg J, Zedenius J, Ekman E, Tennvall J, Blomgren H, Grimelius L, Lundell G \& Wallin G. Anaplastic giant cell carcinoma of the thyroid gland: treatment and survival over a 25-year period. World Journal of Surgery 1998 22 725-730.

25 Venkatesh YS, Ordonez NG, Schultz PN, Hickey RC, Goepfert H \& Samaan NA. Anaplastic carcinoma of the thyroid. A clinicopathologic study of 121 cases. Cancer 199066 321-330.

26 Lo CY, Lam KY \& Wan KY. Anaplastic carcinoma of the thyroid. American Journal of Surgery 1999177 337-339.

27 Hadar T, Mor C, Shvero J, Levy R \& Segal K. Anaplastic carcinoma of the thyroid. European Journal of Surgical Oncology 199319 511-516.

28 O'Neill JP, O'Neill B, Condron C, Walsh M \& Bouchier-Hayes D. Anaplastic thyroid cancer: improved insight and therapeutic stategy into highly aggressive disease. Journal of Laryngology and Otology 2005119 585-591.

Received 15 November 2006

Accepted 29 January 2007 